\title{
Performance Analysis of Variable Bit Rate Multiclass Services in the Uplink of a Dynamic Complete Partitioning Round-Robin Carrier-Hopping Multirate Multi-carrier DS-CDMA System
}

\author{
Tung Chong Wong ${ }^{1}$, Jon W. Mark ${ }^{2}$, and Kee Chaing Chua ${ }^{3}$ \\ ${ }^{1}$ Institute for Infocomm Research, 21 Heng Mui Keng Terrace, Singapore 119613, Singapore \\ wongtc@i2r.a-star.edu.sg \\ ${ }^{2}$ Center for Wireless Communications, University of Waterloo, \\ Waterloo, Ontario, Canada N2L 3G1 \\ jwmarkabbcr. uwaterloo.ca \\ ${ }^{3}$ Electrical and Computer Engineering, National University of Singapore, \\ 10 Kent Ridge Crescent, Singapore 119260, Singapore \\ chuakc@nus.edu.sg
}

\begin{abstract}
An analytical formulation of the outage probability in terms of bit error rate specification for variable bit rate (VBR) multiclass services in the uplink of a dynamic complete partitioning (DCP) round-robin carrier-hopping multirate Multi-Carrier (MC) DS-CDMA cellular system is presented. The analytical framework is formulated for the general case in which different traffic classes have different spreading gains in each of the subcarriers. The analytical work leads to the determination of the capacity region of this cellular system for VBR traffic. Numerical results show that the system capacity with DCP can be larger than that with complete sharing (CS) of the subcarriers.
\end{abstract}

\section{Introduction}

3G/4G cellular mobile networks are expected to support integrated multimedia services with different qualities of service (QoS) for different service classes. Ref. [1] considers $K$ traffic classes in $3 \mathrm{G} / 4 \mathrm{G}$ cellular systems. In [1], the traffic source is assumed to be an on/off process which is valid only for voice or data traffic but not for video or other traffic which can be modeled as a multirate traffic [2]. Ref. [3] considers a multirate traffic model for variable bit rate (VBR) traffic in the uplink of a round-robin carrier-hopping multirate Multi-Carrier (MC) DS-CDMA system with multiclass traffic using complete sharing (CS) of all the subcarriers in the system.

Recently, MC-CDMA systems have been receiving a lot of attention as they can promise high data rate required by $4 \mathrm{G}$ mobile cellular systems and be effective in mitigating multipath fading and rejecting narrowband interference [4]. This paper considers a multirate traffic model for VBR multiclass traffic to study the capacity of the uplink of a dynamic complete partitioning (DCP) round-robin carrier-hopping multirate MC-DS-CDMA system. In traditional resource allocation schemes at the 
connection level, a CS scheme will give lower blocking probabilities than those from a CP scheme as they have "hard capacity." On the other hand, CDMA-based systems have "soft capacity" depending on intra- and inter-cell interference. A salient feature of this paper is the use of DCP to dynamically allocate subcarriers to different traffic classes to eliminate intra- and inter-cell interference from other classes. This helps to increase system capacity. The outages in the subcarriers of each class are minimized by spreading the subcarriers' signal-to-interference ratio evenly among all its subcarriers by using a dynamic round-robin carrier-hopping allocation scheme. This will also help to increase system capacity. The activity factor of a user in a subcarrier is modeled as though it is an on/off source in a single-carrier CDMA system as in [1].

\section{Dynamic Complete Partitioning Round-Robin Carrier-Hopping Multirate MC-DS-CDMA}

A single-carrier DS-CDMA system has a spread-spectrum bandwidth of $W$ at a center frequency of $f$. On the other hand, a multi-carrier DS-CDMA system has a spreadspectrum bandwidth of $W / N_{c}$ at frequency $f_{c}$ in each of its subcarriers, where $N_{c}$ is the total number of subcarriers, $c=1,2, \ldots, N_{c}, N_{c}=\sum_{i=1}^{K} N_{c i}$, and $N_{c i}$ is the number of class $i$ subcarriers. The same spreading code can be used in each of the subcarriers for a user. A VBR source with discrete bit rate levels as modeled in Section 3 is considered. Each level has a bit rate of $R_{i}$, where $i=1,2, \ldots, K$ and $K$ is the number of traffic classes. The highest level is $M_{i}$ and it has a bit rate of $M_{i} R_{i}$. Each level of bit rate is transmitted through one subcarrier. Thus a variable bit rate source is transmitted through a number of subcarriers, each using the same spreading code. Every user in the same class transmits at the same bit rate, $R_{i}$, in each group of subcarriers that are dynamically allocated to that class.

Consider the case of two traffic classes. To evenly spread the usage of the subcarriers and outage probabilities in the subcarriers and to increase the support of the number of users in the system, we propose to use a round-robin carrier-hopping multirate MC-DS-CDMA. This round-robin carrier-hopping subcarrier allocation scheme works as follows. Assume that the first level of bit rate is transmitted first in one of the $N_{c l}$ subcarriers and the other levels of bit rate are transmitted in the next few subcarriers for a class 1 user. Similarly, the first level of bit rate for a class 2 user is transmitted first in one of the $\left(N_{c l}+1\right)$ th to $\left(N_{c}\right)$ th subcarriers. The usages of the subcarriers are shown in Table 1. The symbol, ô, represents that a reference subcarrier is used, while the symbol, o, represents that the subsequent subcarriers are used. The reference subcarrier of each class is moved in a round-robin manner over all its subcarriers. The number of subcarriers dynamically allocated to class 1 users is $N_{c l}$, while that to class 2 users is $N_{c 2}=N_{c}-N_{c 1}$. As shown in Table 1, the goal of evenly spreading the usage of the subcarriers is achieved. Thus the outage probabilities in the subcarriers are lowered and the number of users that can be supported is increased. Furthermore, the complete partitioning of the subcarriers to the different classes is dynamically adjusted to maximize the number of users that can be supported by using the DCP admission 
region curves in Section 4. The DCP admission region can be stored in a lookup table and be referenced to execute the dynamic complete partitioning allocation of subcarriers to each class when DCP can support more users than CS.

Table 1. Subcarriers used for VBR sources in a dynamic complete partitioning round-robin carrier-hopping MC-DS-CDMA system with 2 classes

\begin{tabular}{|c|c|c|c|c|c|c|c|c|c|c|}
\hline \multirow{3}{*}{$\begin{array}{l}\text { Time } \\
\text { epoch }\end{array}$} & \multicolumn{10}{|c|}{ Subcarrier(s) used } \\
\hline & \multicolumn{4}{|c|}{ Class 1} & \multicolumn{6}{|c|}{ Class 2} \\
\hline & 1 & 2 & 3 & $N_{c t}$ & $N_{c l}+1$ & $N_{c}+2$ & $N_{c}+3$ & $N_{c}+4$ & $N_{c c}+5$ & $N$ \\
\hline$t_{1}$ & $\hat{o}$ & & & & $\hat{o}$ & & & . & & \\
\hline$t_{2}$ & & $\hat{o}$ & o & & & ô & o & & & \\
\hline$t_{3}$ & 0 & & ô & o & & & $\hat{o}$ & o & 0 & \\
\hline$t_{4}$ & o & & & $\hat{o}$ & & & & ô & o & \\
\hline$t_{5}$ & ô & & & & & & & & $\hat{o}$ & \\
\hline$t_{6}$ & & $\hat{o}$ & o & & o & & & & & ô \\
\hline$t_{7}$ & o & & ô & o & $\hat{o}$ & o & o & & & \\
\hline
\end{tabular}

\section{Analytical Model}

From [2], a VBR source can be modeled by a continuous-time Markov chain with finite states. Each state represents the discrete level of bit rate generated by a single source. We assume that the highest level is state $M_{i}$, which is also matched to the maximum number of subcarriers used by a class $i$ user. This means that each level has a data rate of $R_{i}$ corresponding to the use one class $i$ spreading code in one subcarrier. Every user uses the same spreading code in its subcarriers. If $M_{i}=1$, the source is an on/off source. Each level can be modeled by a two-state mini-source with increase and decrease rates of $\alpha_{i}$ and $\beta_{i}$, respectively. Thus the continuous-time Markov chain for a single source at state $m$ has increase and decrease rates of $\left(M_{i}-m\right) \alpha_{i}$ and $m \beta_{i}$, respectively. This Markov chain is shown in Fig. 1.

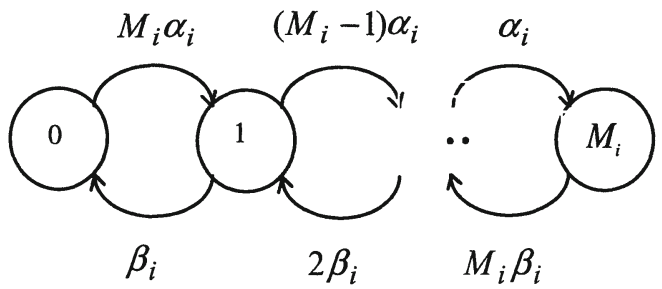

Fig. 1. Continuous-time Markov chain for a single variable bit rate source

The steady-state probability of being in state $m$, denoted by $P_{m}$, is given by 


$$
P_{m}=\left(\begin{array}{c}
M_{i} \\
m
\end{array}\right)\left(p_{i}\right)^{m}\left(1-p_{i}\right)^{M_{i}-m}, m=0,1,2, \ldots, M_{i},
$$

where $p_{i}=\alpha_{i} /\left(\alpha_{i}+\beta_{i}\right)$. The probability that subcarrier $c$ is used for transmission given that the source is in state $m$, denoted by $P_{c \mid m}$, is given by

$$
P_{c \mid m}=m / N_{c i}, m=0,1,2, \ldots, M_{i}, c=1,2, \ldots, N_{c i}, M_{i} \leq N_{c i} \text {. }
$$

Unconditioning the dependence on state $m$, the probability that subcarrier $c$ is used for transmission, denoted by $P_{c}$, is given by

$$
P_{c}=\sum_{m=0}^{M_{i}} P_{c \mid m} P_{m}=\sum_{m=0}^{M_{i}}\left(\frac{m}{N_{c i}}\right)\left(\begin{array}{c}
M_{i} \\
m
\end{array}\right)\left(p_{i}\right)^{m}\left(1-p_{i}\right)^{M_{i}-m}=\frac{M_{i} p_{i}}{N_{c i}}, M_{i} \leq N_{c i} .
$$

Note that if $M_{i}=1$, the source is an on/off source and the probability that subcarrier $c$ is used for transmission is simply equal to the source activity factor, $p_{i}$, divided by its number of subcarriers, $N_{c i}$. This is expected due to the round-robin carrier-hopping allocation scheme used. This allocation scheme evenly spreads the subcarriers' usage by a source. The $E_{b} / I_{0}$ for class $i$ in each of its subcarriers, $\gamma_{i}$, is given by

$$
\gamma_{i}=G_{i} /\left(\sum_{j=1}^{n_{i}-1} \psi_{i j}+\left(I_{i} / S_{i}\right)+\left(\eta / S_{i}\right)\right), i=1,2, \ldots K,
$$

where $\psi_{i j} \in\{0,1)$ is a Bernoulli random variable indicating the activity factor of the spreading code used by the $j$ th user of class $i$ in each of its subcarriers. The probability that an active spreading code in a subcarrier is used by source $i$ is given by $\operatorname{Pr}\left[\psi_{i j}=1\right]=q_{i}=P_{c}, i=1,2, \ldots, K . G_{i}=\left(W / N_{c i}\right) / R_{i}$ is the class $i$ processing gain in subcarrier $c$. In the denominator of (4), $n_{i}$ is the number of class $i$ users, the first term is due to the intra-cell interference in subcarrier $c$ from other users in class $i$, the second term is due to the inter-cell interference power, $I_{i}$, in the subcarrier from users from the same class, where $S_{i}$ is the received power in the subcarrier for a class $i$ user, and the last term is due to background noise power, $\eta$, in the subcarrier. Note that by using the proposed DCP scheme, the intra- and inter-cell interference in subcarrier $c$ from other classes are eliminated.

Treating each subcarrier as orthogonal from other subcarriers, we can model each subcarrier as though it has on/off multiclass traffic with their multirate MC-DSCDMA sources' subcarrier activity factors being treated like the on/off sources' activity factors. Thus we can make use of the approach and results in [1]. From [1], the inter-cell interference-to-signal ratio in subcarrier $c$ for a class $i$ user is given by

$$
I_{i} / S_{i}=\left(r_{m} / r_{d}\right)^{4} 10^{\left(\varepsilon_{d}-\varepsilon_{m}\right) / 10}
$$

where $r_{d}$ is the distance between the inter-cell mobile that is causing interference and the intra-cell base station, $r_{m}$ is the distance between the inter-cell mobile and its own base station, and $\varepsilon_{d}$ and $\varepsilon_{m}$ are Gaussian random variables with zero mean and standard deviation $\sigma$. Since $\varepsilon_{d}$ and $\varepsilon_{m}$ are independent, $\left(\varepsilon_{d}-\varepsilon_{m}\right)$ is a Gaussian random variable with zero mean and $2 \sigma^{2}$ variance.

The mean and variance of $I_{i} / S_{i}$ are upper bounded by [1]

$$
E\left[I_{i} / S_{i}\right]=q_{i} \rho_{i} \iint f\left(r_{m} / r_{d}\right) d A \leq \mu_{i i},
$$

and

$$
\operatorname{Var}\left[I_{i} / S_{i}\right] \leq \rho_{i} \iint\left[q_{i} g\left(r_{m} / r_{d}\right)-q_{i}^{2} f^{2}\left(r_{m} / r_{d}\right)\right\} d A=\sigma_{i i}^{2},
$$


where $\mu_{i i}$ is the upper bound on the mean of $I / S_{i}, \sigma_{i i}^{2}$ is the upper bound on the variance of $I / S_{i}, \rho_{i}=2 n_{i} / \sqrt{3}$ is the density of class $i$ users per unit area

$$
\begin{aligned}
& f\left(r_{m} / r_{d}\right)=\left(r_{m} / r_{d}\right)^{4} e^{(\sigma \ln 10 / 10)^{2}}\left[1-Q\left(40 \log \left(r_{d} / r_{m}\right) / \sqrt{2 \sigma^{2}}-\sqrt{2 \sigma^{2}} \ln 10 / 10\right)\right], \\
& g\left(r_{m} / r_{d}\right)=\left(r_{m} / r_{d}\right)^{8} e^{(\sigma \ln 10 / 5)^{2}}\left[1-Q\left(40 \log \left(r_{d} / r_{m}\right) / \sqrt{2 \sigma^{2}}-\sqrt{2 \sigma^{2}} \ln 10 / 5\right)\right],
\end{aligned}
$$

and $Q(y)=\int_{y}^{\infty} e^{-x^{2} / 2} d x / \sqrt{2 \pi}$.

Let $B E R_{i}^{*}$ denote the BER requirement in a subcarrier for class $i$ users and $S I R_{i}^{*}$ denote the SIR requirement in a subcarrier for class $i$ users. The system capacity is defined as the maximum $\left(n_{1}, \ldots, n_{i}, \ldots, n_{K}\right)$ that can be supported such that the achieved SIR in a subcarrier is greater than or equal to the required $S I R_{i}^{*}$ in the subcarrier $99 \%$ of the time for all classes. That is, the outage probability in a subcarrier is defined as

$$
\operatorname{Pr}\left[B E R_{i} \geq B E R_{i}^{*}\right]=\operatorname{Pr}\left[S I R_{i} \leq S I R_{i}^{*}\right]=\operatorname{Pr}\left[\sum_{j=1}^{n_{i}-1} \psi_{i j}+\left(I_{i} / S_{i}\right) \geq \delta_{i}\right]
$$

where $\delta_{i}=\left(G_{i} / \gamma_{i}\right)-\left(\eta / S_{i}\right), i=1,2, \ldots, K$. Invoking the central limit approximation and solving (10), we have

$$
\begin{aligned}
\operatorname{Pr}\left[B E R_{i} \geq B E R_{i}^{*}\right] & =\sum_{l_{i}=0}^{n_{i}-1} \operatorname{Pr}\left[\frac{I_{i}}{S_{i}} \geq \delta_{i}-l_{i} \mid \sum \psi_{i j}=l_{i}\right] \operatorname{Pr}\left[\sum \psi_{i j}=l_{i}\right] \\
& =\sum_{l_{i}=0}^{n_{i}-1} Q\left(\frac{\delta_{i}-\mu_{i}}{\sigma_{i}}\right)\left(\begin{array}{c}
n_{i}-1 \\
l_{i}
\end{array}\right) q_{i}^{l_{i}}\left(1-q_{i}\right)^{n_{i}-1-l_{i}},
\end{aligned}
$$

where $\mu_{i}=l_{i}+\left(q_{i} \rho_{i} / q_{1} \rho_{1}\right) \mu_{11}$, and $\sigma_{i}=\sqrt{\sigma_{i i}^{2}}, \quad i=1,2, \ldots, K$. The analysis here can be used to determine the system capacity with the proposed DCP scheme for allocation of subcarriers in 4G MC-DS-CDMA mobile systems.

Table 2. Parameter Values Used

\begin{tabular}{|c|l|c|l|}
\hline Symbol & Value & Symbol & \multicolumn{1}{|c|}{ Value } \\
\hline$N_{c}$ & 10 or 20 & $G_{i}$ & $\left(W / N_{c i}\right) / R_{i}$ \\
\hline$M_{1}$ & 1 & $B E R_{1}^{*}=B E R_{2}^{*}$ & $10^{-3}$ \\
\hline$M_{2}$ & 3 & $\sigma$ & $8 \mathrm{~dB}$ \\
\hline$\alpha_{1}$ & $1 / 0.625$ & $\gamma_{1}=\gamma_{2}$ & $7 \mathrm{~dB}$ \\
\hline$\beta_{1}$ & $1 / 0.375$ & $S_{1} / \eta$ & $-1 \mathrm{~dB}$ \\
\hline$\alpha_{2}$ & 0.9 & $S_{2} / \eta$ & $\left(S_{1} / \eta\right) \times\left(S_{2} / S_{1}\right), \frac{S_{2}}{S_{1}}=\left(\frac{G_{1}}{\gamma_{1}}+q_{1}\right) /\left(\frac{G_{2}}{\gamma_{2}}+q_{2}\right)$ \\
\hline$\beta_{2}$ & 0.1 & $\mu_{11}$ & $0.0247 n_{1}$ or $0.01235 n_{1}$ \\
\hline$R_{1}=R_{2}$ & $80 \mathrm{kbps}$ & $\sigma_{11}^{2}$ & $0.01256 n_{1}$ or $0.00641 n_{1}$ \\
\hline$W$ & $50 \mathrm{MHz}$ & $\sigma_{22}^{2}$ & $0.01256 n_{2} q_{2} / q_{1}$ or $0.00641 n_{2} q_{2} / q_{1}$ \\
\hline
\end{tabular}




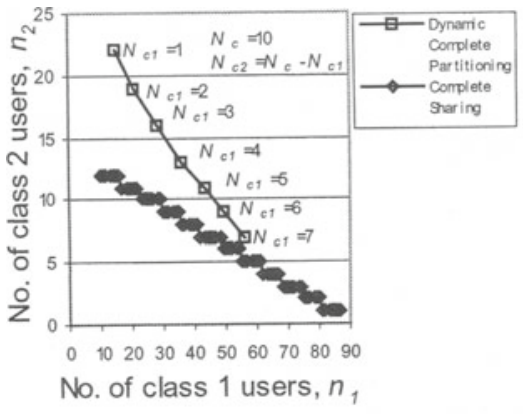

(a)

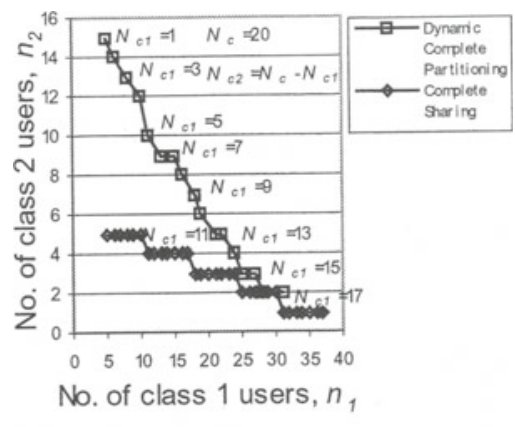

(b)

Fig. 2. System capacity with (a) $N_{c}=10$, and (b) $N_{c}=20$

\section{Numerical Results}

In this section we present results for the system capacity with 2 classes $(K=2)$ as illustrative examples. The parameter values used in the numerical examples are tabulated in Table 2. The admissible regions for the system capacities for CS and DCP with $N_{c}=10$ and $N_{c}=20$ are shown in Fig. 2. The results for CS are obtained using the analytical formulation in [3]. Fig. 2 shows that the system capacities, $\left(n_{1}, n_{2}\right)$, of DCP can be larger than those of CS, with these numbers of subcarriers. Admission to the system is possible only when the elements of the admitted doublet $\left(n_{1}, n_{2}\right)$ are on or below these curves. Furthermore, the system capacities of CS and DCP for $N_{c}=10$ is larger than those for $N_{c}=20$. Note that the probability that an active spreading code in a subcarrier is used by source $i, q_{i}$, is increased by having a smaller number of subcarriers to use, $N_{c i}<N_{c}$, (see (4)) by using the DCP scheme for allocation of subcarriers to different classes. However, this is significantly offset, for low to above average values of $N_{c 1}$, by eliminating the intra- and inter-cell interference from other classes in its own subcarriers. The admission region can be stored and retrieved from a look-up table to perform DCP of subcarriers to different classes when the number of users to be supported is larger than that of CS. The DCP round-robin carrier-hopping multirate MC-DS-CDMA cellular system introduced in this paper shows great promise in terms of capacity usage for multimedia services.

\section{References}

1. Wong, T.C., Mark, J.W., Chua, K.C., Yao, J., Chew, Y.H.: Performance Analysis of Multiclass Services in the Uplink of Wideband CDMA. IEEE ICCS (2000) 692-696

2. Maglaris, B., Anastassiou, D., Sen, P., Karlsson, G., Robbins, J.D.: Performance Models of Statistical Multiplexing in Packet Video Communications. IEEE Trans. on Comm., Vol. 36, No. 7, (1988) 834-844 
3. Wong, T.C., Mark, J.W., Chua, K.C.: Performance Analysis of Variable Bit Rate Multiclass Services in the Uplink of a Round-Robin Carrier-Hopping Multirate Multi-Carrier DSCDMA System. International Conference on CIC, (2003) CD-ROM

4. Simon, M.K., Alouini, M.-S.: Digital Communication over Fading Channels: A Unified Approach to Performance Analysis. John Wiley and Sons (2000) 